KINETIK, Vol. 3, No. 2, May 2018, Pp. 153-162

ISSN :2503-2259

E-ISSN :2503-2267

153

\title{
Comparative Analysis of Tracking Objects Using Optical Flow and Background Estimation on Silent Camera
}

\author{
Wahyu Supriyatin ${ }^{* 1}$, Winda Widya Ariestya ${ }^{2}$, Ida Astuti ${ }^{3}$ \\ 1,2,3Universitas Gunadarma/Faculty of Computer Science and Information Technology \\ ayu_ws@staff.gunadarma.ac.id ${ }^{*}$, winda_widya@staff.gunadarma.ac.id ${ }^{2}$, \\ astuti@staff.gunadarma.ac.id ${ }^{3}$
}

\begin{abstract}
Tracking and object is one of the utilizations on the field of the computer vision application. Object tracking utilization as a computer vision in this study is used to identify objects which exist within a frame and calculate the number of objects passing within a frame. The utilization of computer vision in various fields of application can be used to solve the existing problems. The method used in object tracking is by comparison between optical flow estimation method with background method. The test is conducted by using a still camera for both methods by making changes to the parameter values used as a reference. The results of the tests, conducted on the three video objects by comparing the two methods show a Total Recorded Time better than those of the background estimation method, being smaller than 100 seconds. Testing both methods successfully identifies the object tracking and calculates the number of passing cars.
\end{abstract}

Keywords: Analysis, Background Estimation, Computer Vision, Object Tracking, Optical Flow

\section{Introduction}

Tracking objects is a form of application of computer vision applications. Some areas of life use object tracking to solve a problem, such as monitoring, vehicle navigation and robot navigation. The process of vision which was originally performed by human vision will be replaced by using computers. Quick object movement becomes invisible to capture by the human eyes. The purpose of the object tracking is to associate the tracking target object in a respective video frame [11]. Object tracking can be performed using object feature extraction and detect objects in a sequence of frames [2].

Computer vision is an automated process of interpreting large number of processes for visual perception, such as acquisition, image processing, classification, recognition and decision making [3]. Computer vision techniques consist of characteristic of object estimation in the image. The measurements are related to object geometry and interpret the object geometry information [3].

Optical flow of video frames is a vector field which moves pixels or sub pixels. Optical flow method uses estimation techniques of optical flow to obtain the motion vector estimation in each frame of a video sequence. Hence, the moving object detected by the program block is converted into a binary image. This is executed by applying thresholding and analysis completed with morphological operations on each image in the foreground [4].

Techniques used in the method background estimation are segmentation motion detection in the image area or region engaged by outlining the image based on pixels with pixels from the image background flattened by drawing initialization [5]. The working principle of this method is to initialize the background first then detects the frame where a moving object is located. The frame then will be reduced by the background frame to detect moving object [5]. The background estimation method is sensitive to any changes in the external environment. Therefore, it only applies to the conditions with the familiar environmental background.

The purpose of this research is to conduct a comparative analysis to perform object tracking between two methods, optical flow method and background estimation method. Object tracking research is conducted in the form of object identification of the passing cars with background and counts the number of passing cars in a frame. Videos used in the study are taken by using a still camera. A video object is analyzed in comparison by using the parameter values in each frame either the current value of default condition or the current value in testing condition. The results of tests performed on the video of parameter values will be analyzed for comparison between the

Supriyatin, W., Ariestya, W., \& Astuti, I. (2018). Comparative Analysis Tracking Objects Using Optical Flow and Background Estimation on Silent Camera. Kinetik, 3(2). doi:http://dx.doi.org/10.22219/kinetik.v3i2.594

Receive November 29, 2017; Revise December 15, 2017; Accepted January 02, 2018 
two methods in terms of both the number of passing cars and cars successfully counted, identified object in frame, video separation between the object and the background (object threshold result) and the simulation results in Matlab Simulink Profile Report between the two methods used.

There are some previous researches on the utilization of computer vision in object tracking method to calculate the speed of passing vehicles, counting the number of vehicles passing per second or monitoring traffic congestion on the road.

Comparative research on object tracking of the passing cars passes using static camera with the camera moving in the default parameter and changed the parameter values using the optical flow method [6][7]. The results obtained in the study conducted by tracking default parameter values is more accurate compared with the altered parameter values both in static and moving cameras.

Shukla and Saini (2015) in a study entitled "Moving Object Tracking of Vehicle Detection: A Concise Review" detected objects in video, and motion tracking was used to identify the characteristics which appear in the image processing domain and computer vision [1]. The traffic image analysis conducted in this paper is the traffic analysis, vehicle motion detection, segmentation approach and vehicle tracking approach. The information on temporal feature and motion tracking is used to identify the vehicle, identifying the vehicle shape, color, and type [8]. Optical flow method and background subtraction technique can help to determine the vehicle speed based on video sequence frames.

Singh and Sharma (2015) in their research utilized Simulink model for object tracking using optical flow [9]. Object tracking is conducted to estimate the location, speed and distance parameters of the moving objects with the aid of a silent camera. The study was conducted by using Lukas-Kanade algorithm used to detect moving objects by altering the intensity of the frame. The test system can remove unwanted object motions in a vehicle other than a sequence of image frames by using multiple conditions.

In 2015, according to Vinary and Kumar's study entitled "Object Tracking Algorithm Using Background Subtraction," it stated that identifying moving objects from a sequence video is fundamental and the most important application in computer vision [5]. The experimental results in this study are the clarity of the image obtained using the elimination background technique is better than using background registration technique. The experiments performed show accuracy in counting vehicles detected approximately $94 \%$.

The study by Scott, Pusateri and Cornish entitled "Kalman Filter Based Video Background Estimation" explained that background estimation using Kalman's filtration provides information about the full background to be used without any user intervention [10]. The design of this study provides an effective method for efficient foreground extraction from computer vision. Onedimensional Kalman filtration is applied to each pixel of the camera array to track the intensity of the pixel.

The study entitled "Optical Flow Based Moving Object Detection and Tracking for Traffic Surveillance" by Aslani and Nasab (2013) discusses the system which was developed to gather useful information from silent cameras to detect moving objects in digital video [2]. To eliminate noise, the median filter was used and the unused objects were removed by thresholding algorithm in the morphological operations.

Revathi and Hemalatha in "Certain Approach of Object Tracking using Optical Flow Techniques" (2012) studies on object tracking by optical flow method using Bayesian algorithm on detected objects in each frame as feature extraction method [4]. The distance covered by the object was determined by the pixel mass by using Euclidean distance formula. The object's speed was calculated by finding a moving object in the distance with the sequence and video frame rate.

Mahamuni, Patil and Thakar (2014) in a study entitled "Moving Object Detection Algorithm using Background Subtraction using Simulink" stated that the method used to combine statistics with moving objects acquired knowledge in the processing of the frame [11]. Previously, approach exploits were used to subtract the background object to improve the object segmentation. Algorithm approach of background subtraction can be fast, flexible and precise in terms of pixel accuracy.

\section{Research Method}

The method used in this research is optical flow method and background estimation method. These two methods in this study will be run and tested by using the tools on Matlab Simulink. Optical flow method used in the study is the Horn-Schunck algorithm. Figure 1 is a Horn-

KINETIK Vol. 3, No. 2, May 2018: 153-162 
Schunck algorithm used to perform object tracking using Matlab Simulink. Horn-Schunck algorithm with its advantages can generate a high density of flow vectors. The information flow can be lost in the internal part of a homogeneous object filled with motion boundary. However, it has drawbacks to be more sensitive to noise than local method [4].

Optical flow method offers some advantages, among others, in producing motion vectors with high population density. The existing disadvantages of optical flow method are being very sensitive to noise, having a large amount of calculation, being sensitive to sound and being inappropriate or difficult to object detection in real time [9]. Optical flow method using a target motion vector of characteristics may vary with time to detect the moving areas in a sequence of images [5].

Figure 2 presents an object tracking algorithm with method background estimation using Matlab Simulink. Background estimation method is a method for obtaining a background image with a manual operator capture-based on background image content [11]. The weakness of background estimation method compared to other operators in this method affects greatly because any significant detection can result in the foreground, not the background. Background identification method used is background estimator consists of a temporal median estimator, the temporal median, and background based motion estimator. Object identification of object tracking with the segment and the filter region can form the object in the frame.

The testing results with Matlab Simulink tools in the second method are used to produce the video original form from the input, video motion vector and video background in the form of video of the separation object tracking, the video threshold in the form of video segmentation, video results in the form of video of the object tracking to find out the number of objects identified.

\section{Results and Discussion}

Analysis of object tracking is used to detect the presence of objects in a frame and calculates how many objects, in this case, the passing cars. Object tracking analysis in this research is conducted by using programming language using Matlab tools. Matlab Simulink object tracking analysis is conducted by comparing the two methods, optical flow method (Horn-Schunck algorithm) with Background Estimation method.

Video objects used in the study consist of three pieces, obtained from Matlab library and obtained by recording directly in Bogor and Depok. The third video used as object tracking research is taken by using a silent camera placed at the designated spot to record the movement of the passing cars from the front. Three videos used in the research object tracking in both good methods optical flow and background estimation with a silent camera are as follows:

1. Video Viptraffic.avi (Matlab Library)

2. Video Bogor.avi (Science Direct)

3. Video Depok.avi (Science Direct)

Video used as object tracking are altered its parameter value of each method. The parameter values are used as a reference for testing the optical flow method and the estimation background method either with default values conditions or with the altered conditions as the test value as shown in Table 1.

The tests carried out by performing the addition and subtraction sum parameter value from the condition the default in both methods. Table 2 is the value of the parameter in the default and trials on both the methods used.

The research object tracking using stationary cameras is conducted by the comparison between the default parameter values and the trial parameter values. The test results of the parameter values on the two methods are treated optical flow method and the background estimation method. Table 3 is a table of comparative

The analysis of parameter values is used for both methods on the silent camera. The testing is performed using the parameter values of Matlab Simulink tools with optical flow method using Horn-Schunck algorithm and background estimation method. From testing the two methods on the still camera, it shows the current parameter value in the default and the current conditions in the test parameter values capturing objects which pass and is calculated the number of the passing objects.

The tests conducted on the third video objects use Matlab Simulink tools by using optical flow and estimation background tracking objects presented in Table 4 and Table 5. Table 4 shows the test results of Matlab Simulink to track an object, in this case, the object car with the condition of parameter default values. With parameter default values, to the video library, Matlab

Comparative Analysis Tracking Objects Using Optical Flow and Background Estimation... Wahyu Supriyatin, Winda Widya Ariestya, Ida Astuti 
(Viptraffic.avi) can be obtained both for the car object identification optical flow method and background estimation method. In addition, many passing cars can also be detected in number as well as between the background and the object separated. For videos recorded live both for Depok.avi and Bogor.avi videos, the object can be detected and the car background can also be separated with the object. The threshold on both videos cannot be distinguished between the object with the background so that all the results of the legible threshold. The number of passing cars on the result for both methods can be calculated if it has been read and passed the white line position.

Table 5 presents a table of object tracking test on the silent camera using the trial parameter values. From the testing conducted using optical flow method and background estimation, the large number of cars which pass can be calculated. The number of passing cars calculated as it passes through white line boundary. In the result, the number of passing cars is calculated from the digit 0 and so on, every passing car will be recalculated starting from the number 0 . For method background estimation between the object with the background can be separated, only Depok.avi and Bogor.avi video results of the background, there is little noise to the car object. As for the threshold, it does not only contain objects but also other detected the surrounding objects than car.

The results of the profile report on Matlab Simulink for each good optical flow method and the estimation background method on the silent camera are presented shown in Table 6. Each duration of time spent in the third simulation video tracking is about 45 seconds. From the time comparison, it uses an optical flow method having better Total Recorded Time value because of its smaller value than that of the background estimation method. The duration time of 45 seconds for the method the optical flow has a smaller Total Recorded Time value showing less than around 100 seconds. Meanwhile, the method background estimation has a Total Recorded Time value of more than 100 seconds.

Table 1. Test Parameter Value

\begin{tabular}{ll}
\hline \multicolumn{1}{c}{ Optical Flow Method } & Background Estimation Method \\
\hline a. Total background frame & a. Minimum object area \\
b. Smoothness Factor & b. Maximum object area \\
c. Maximum iteration & $\begin{array}{l}\text { c. Background estimator } \\
\text { (temporal median estimator) }\end{array}$ \\
& $\begin{array}{l}\text { d. Background estimator } \\
\text { (temporal median) }\end{array}$ \\
\hline
\end{tabular}

Table 2. Comparison of Testing Parameter Value of Object Tracking

\begin{tabular}{|c|c|c|c|c|}
\hline \multirow[b]{2}{*}{ Parameter Value } & \multicolumn{2}{|c|}{ Optical Flow Method } & \multicolumn{2}{|c|}{$\begin{array}{l}\text { Background Estimation } \\
\text { Method }\end{array}$} \\
\hline & $\begin{array}{c}\text { Default } \\
\text { Parameter Value }\end{array}$ & $\begin{array}{l}\text { Test Parameter } \\
\text { Value }\end{array}$ & $\begin{array}{l}\text { Default } \\
\text { Parameter } \\
\text { Value }\end{array}$ & $\begin{array}{c}\text { Test } \\
\text { Parameter } \\
\text { Value }\end{array}$ \\
\hline $\begin{array}{l}\text { Total background } \\
\text { frame }\end{array}$ & 1 & $1,5,10$ and 20 & & \\
\hline Smoothness factor & 1 & $1,4,10$ and 20 & & \\
\hline Maximum iteration & 1 & $10,30,50$ and 60 & & \\
\hline $\begin{array}{c}\text { Minimum object } \\
\text { area }\end{array}$ & & & 200 & $\begin{array}{l}100 \text { and } \\
500\end{array}$ \\
\hline $\begin{array}{c}\text { Maximum object } \\
\text { area } \\
\text { Background }\end{array}$ & & & 2500 & $\begin{array}{c}1,000 \text { and } \\
6,000\end{array}$ \\
\hline $\begin{array}{l}\text { estimator (temporal } \\
\text { median estimation } \\
\text { Background }\end{array}$ & & & 2 & 4 and 20 \\
\hline $\begin{array}{l}\text { estimation (temporal } \\
\text { median) }\end{array}$ & & & 30 & 60 and 90 \\
\hline
\end{tabular}

KINETIK Vol. 3, No. 2, May 2018: 153-162 
Table 3. Comparative Analysis of Test Parameter Value on the Silent Camera

Test Result of Optical Flow Method

1. The results of motion vector show cars as the only captured objects.

Default

Parameter

Value

Test

Parameter

Value
2. The results of the threshold show cars looking just emerging as illustrated with white stripes.

3. The results of the application can show the number of passing cars (the number of cars calculated).

1. The results of motion vector capture not only cars but also other surrounding objects such as trees, road dividers, road markings and motors.

2. The results of motion vector capture not only cars but also other surrounding objects but also an embankment, trees, bike and road markings influenced by the number of background frames having more than 1 as illustrated by the white line.

3. The results of the application can show the number of the passing cars (the number of cars calculated) and motors which are calculated as a car with certain conditions.
Test Result of Background Estimation

Method

1. The result show separation between the background object with the car object (for video from Matlab library) and can generate a threshold of car objects.

2. For videos directly recorded using the silent camera (Bogor and Depok videos), a car with an object background can be separated. However, when the threshold is completed, all the objects in the video are captured.

3. The number of passing cars can be captured and calculated by the boundary value of white line position defined either alone or recorded video from the Matlab library.

1. The background can separate the object from the object car (for video from the Matlab library) and produce car threshold to be an object.

2. For videos directly recorded using the silent camera (Bogor and Depok videos), car object can be separated from the background, but there is little noise on the car object. For the results of threshold, all objects in the video are caught and identified instead of just only cars.

3. The number of passing cars can be captured and calculated by the boundary value of white line position defined either alone or recorded videos from the Matlab library.

\section{Tracking Cars Using Optical Flow}

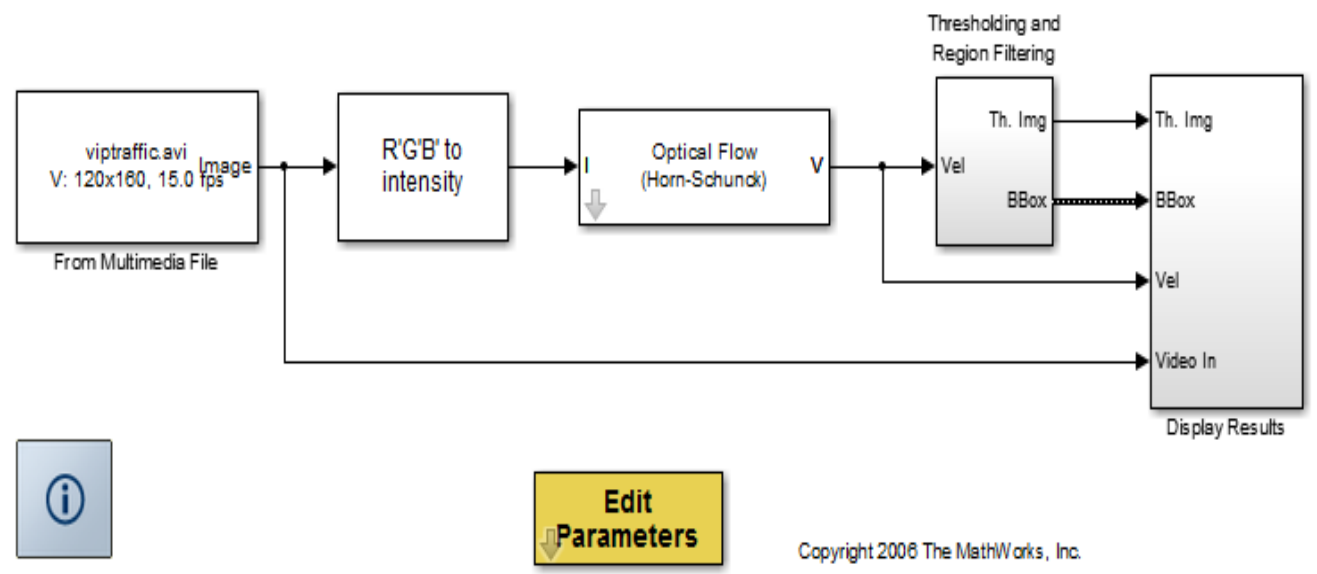

Figure 1. Algorithm of Object Tracking Using Optical Flow Method

Comparative Analysis Tracking Objects Using Optical Flow and Background Estimation...

Wahyu Supriyatin, Winda Widya Ariestya, Ida Astuti 


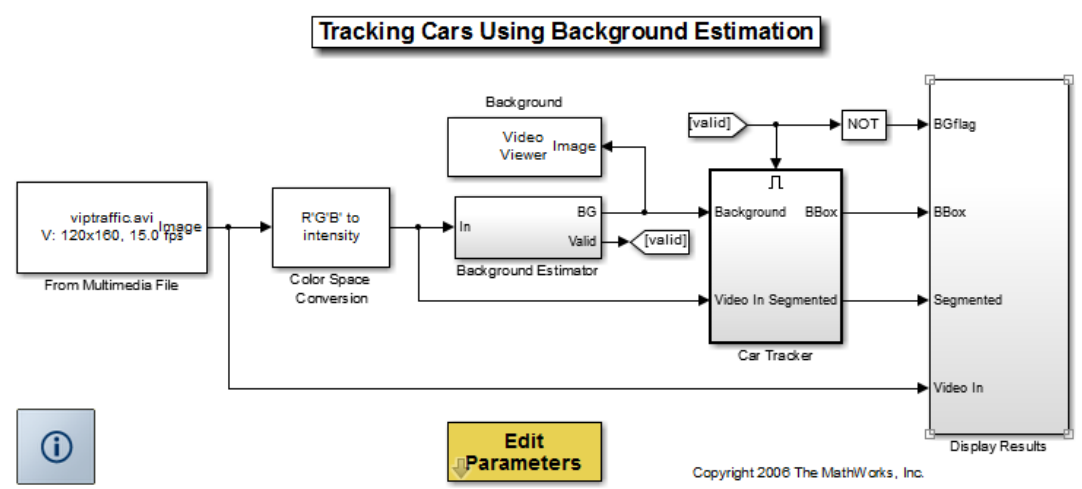

Figure 2. Algorithm of Objects Tracking Using Background Estimation Method

Table 4a. Test Results of Object Tracking on the Silent Camera Using Default Parameter Value Name of the Testing Results of Optical Flow Testing Result of Background Estimation

Video

Viptraffic.avi
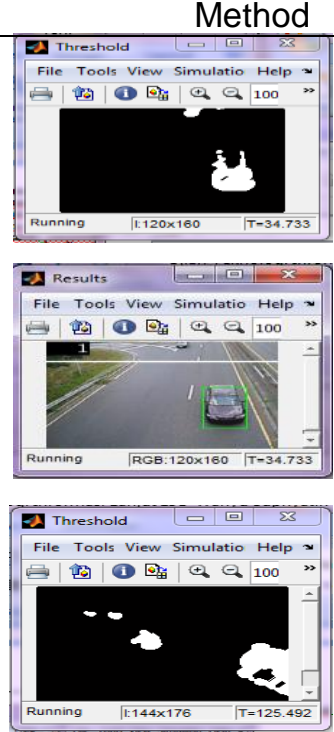

Bogor.avi

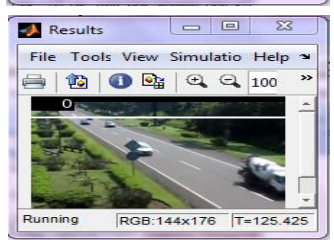
Method
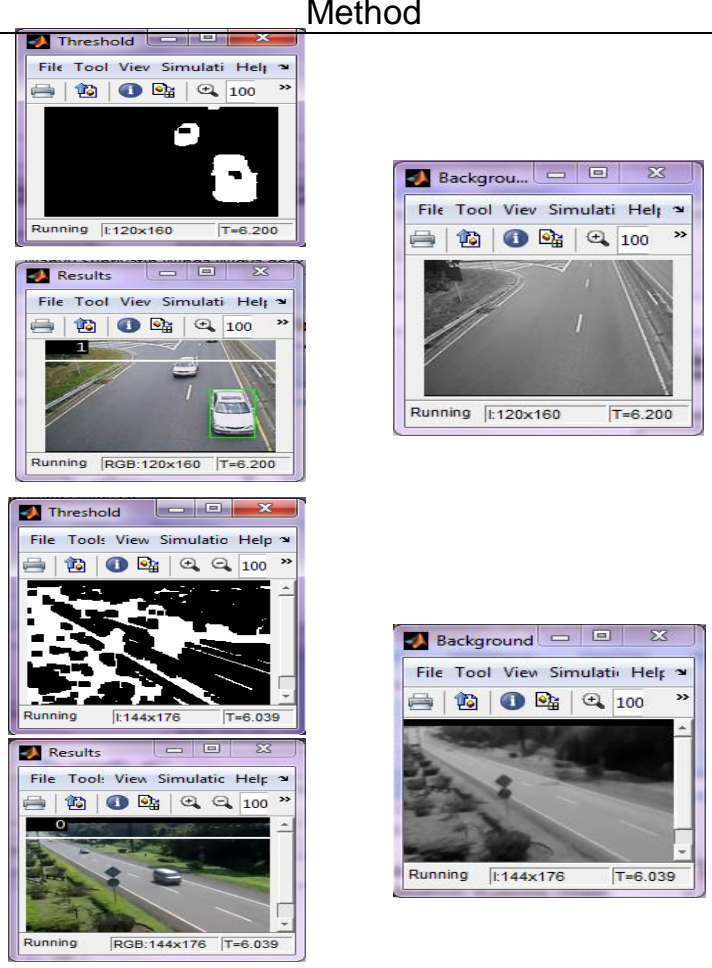

Table 4b. Test Results of Object Tracking on the Silent Camera Using Default Parameter Value Name of Testing Results of Optical Flow Testing Result of Background Estimation the Video Method Method

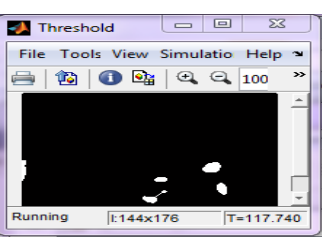

Depok.avi

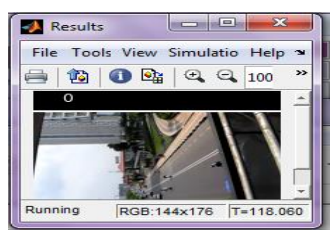

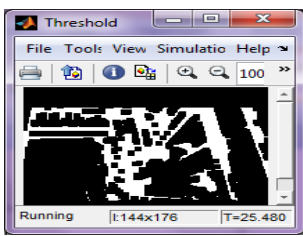

Results

File Tool: Vien Simulatic Helf $=$

File Tool: vien Simulatic HelF

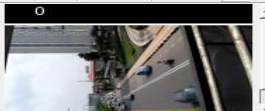

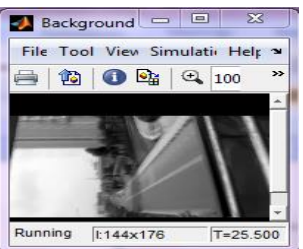

KINETIK Vol. 3, No. 2, May 2018: 153-162 
Table 5a. Test Result of Object Tracking on the Silent Camera Using Test Parameter Value Name of Testing Results of Optical Testing Result of Background Estimation the Video Flow Method Method

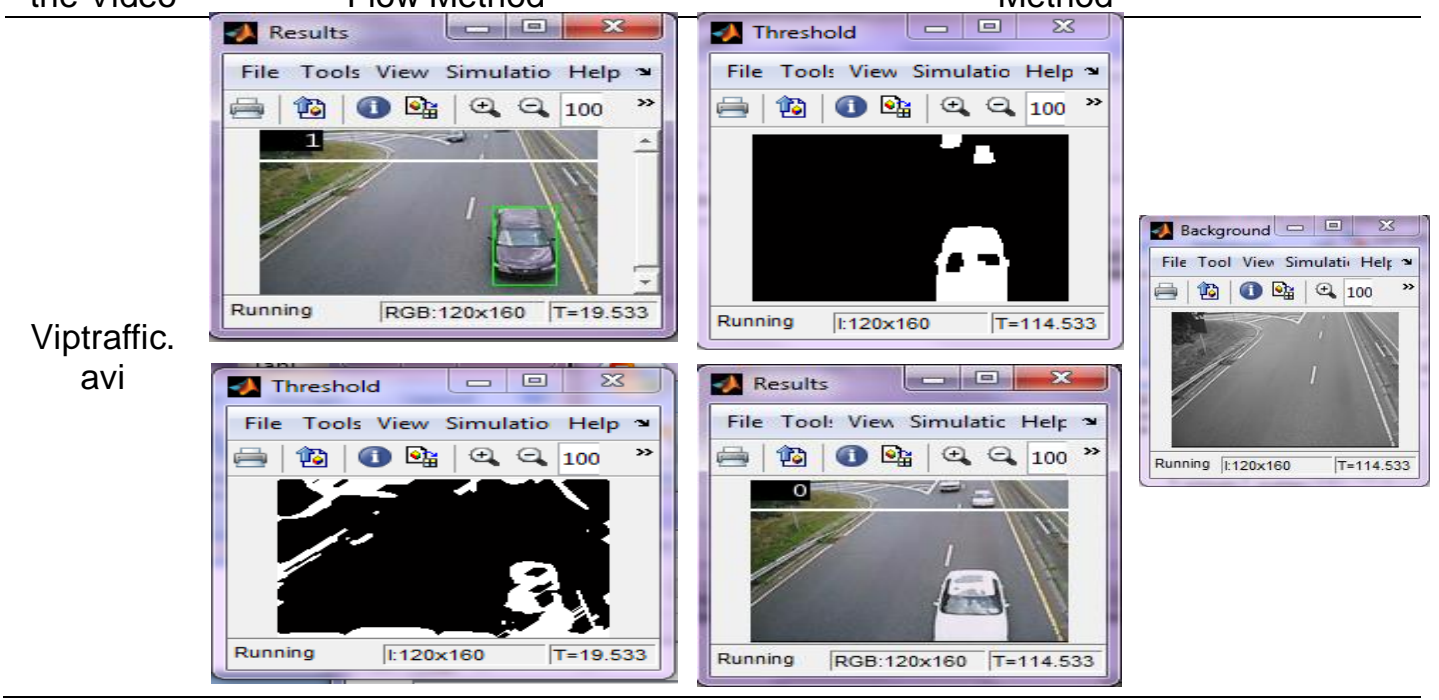

Table 5b. Test Result of Object Tracking on the Silent Camera Using Test Parameter Value Name of Testing Results of Optical Flow Testing Result of Background Estimation the Video Method Method

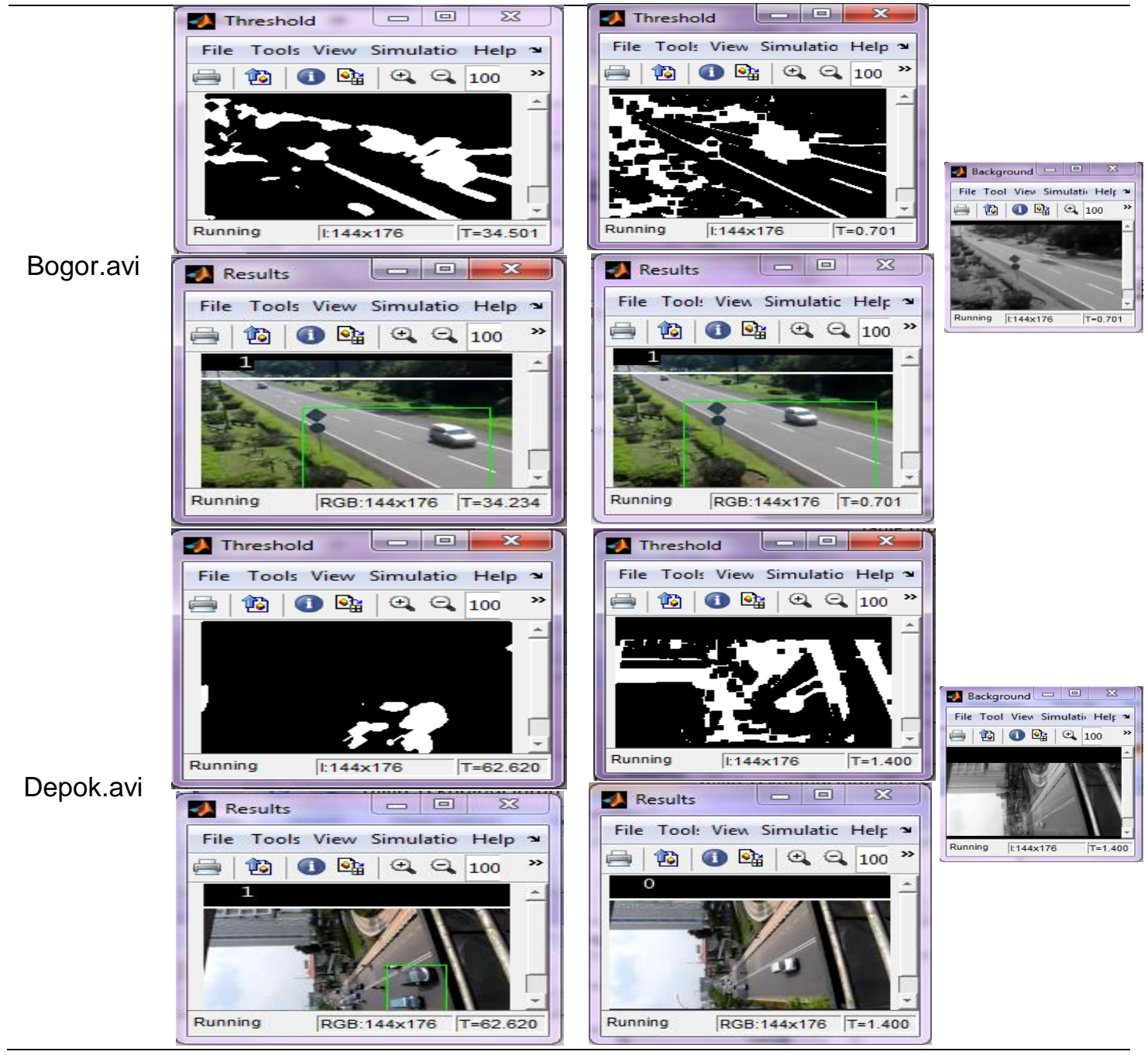

Comparative Analysis Tracking Objects Using Optical Flow and Background Estimation... Wahyu Supriyatin, Winda Widya Ariestya, Ida Astuti 
Table 6. Results of Simulink Profile Report of Object Tracking on Silent Camera

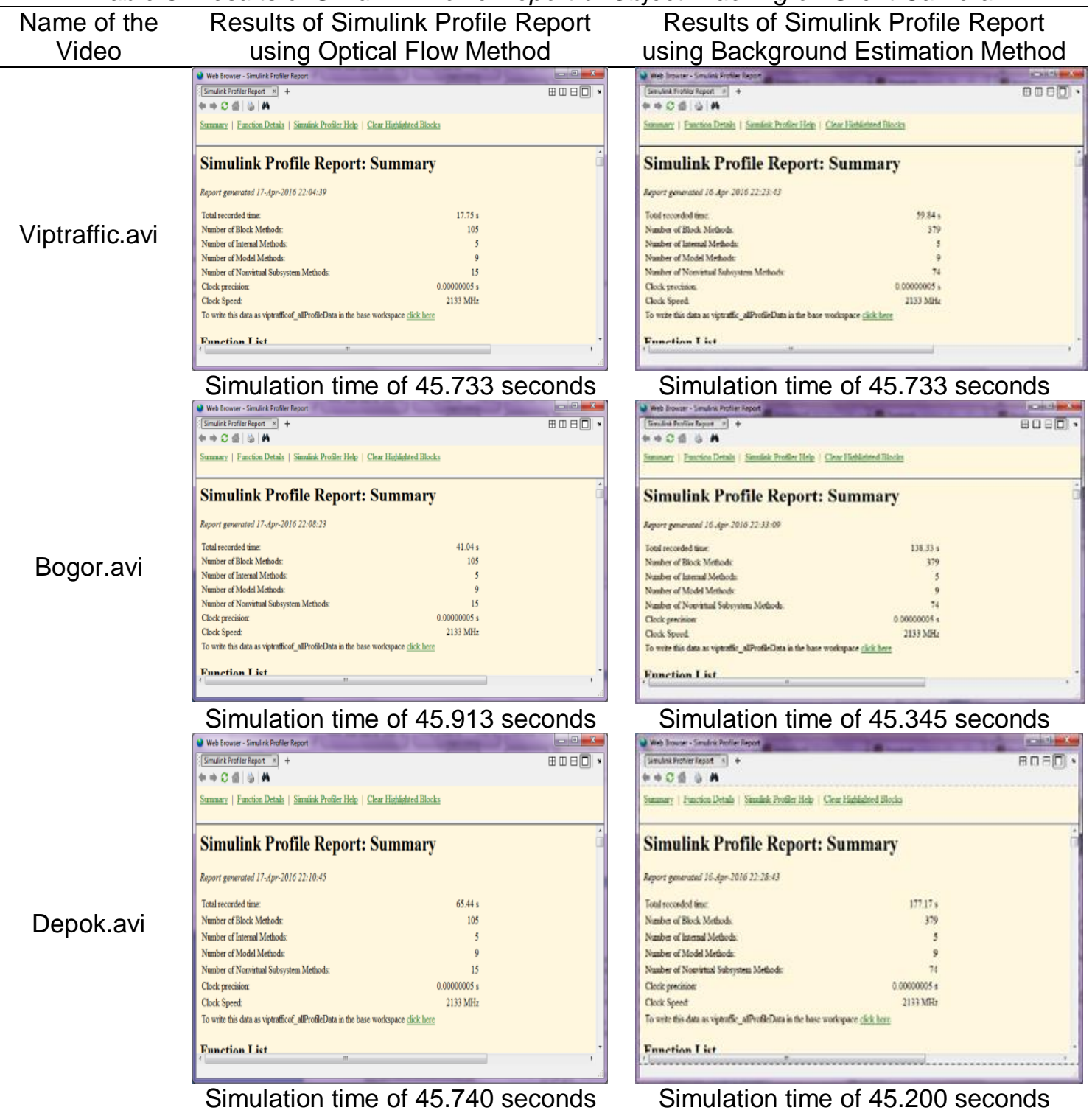

\section{Conclusion}

Object tracking is tracking to calculate the number of passing objects within a frame. This research tracks object such as calculating the number of passing cars in a frame as well as identifying the shapes of passing cars. Car tracking in this research is conducted by doing a comparison between the two methods, the optical flow method (Horn-Schunck algorithm) and background estimation method. The videos used as the objects of the study consist of three pieces. The videos analyzed in the study are taken by using a silent camera. The analysis of object tracking test is carried out by comparing the value of the parameters used, either in a condition of default parameter value or in the test parameter values.

Tracking car objects in this study are employed by using a silent camera on the two methods of using the parameter values as a reference which successfully identify the form of a passing car. It also can calculate the number of cars crossing the white boundary line starting from the number 0 and does not apply to multiplication. Optical flow method can identify the shape of objects and calculate the passing objects. Meanwhile, based on background estimation method, only the videos in the Matlab library manage to be identified their object shapes. The objects with the background can be separated and calculated with the number of passing objects. However, videos recorded directly between the object with the background cannot be separated due to its whole detection. 
The results of Simulink profile report the same simulation time duration of 45 seconds. Optical flow method has better Total Recorded Time than background estimation method. The tests using the silent camera presents smaller value generated by using optical flow accounted as less than 100 seconds when compared with background estimation method which is calculated more than 100 seconds.

Further development for object tracking can identify the object with more detail. Hence, it may distinguish between objects located in the background. On the other hand, it cannot perform calculations in the number of objects in multiple passing cars which do not start from 0 . It can only identify the car object captured as its main targets while other unnecessary objects remain visible.

\section{References}

[1] W. Supriyatin, and W. W. Ariestya, "Analysis of Object Tracking Using Optical Flow in Static and Moving Camera," National Seminar of Information Technological (SRITI), Vol. VIII, Pp. 48-56, 2016.

[2] W. Supriyatin, Y. Rafsyam, and Jonifan, "Analysis of Object Tracking Using Background Estimation in Static and Moving Camera," Orbith, Vol. 13, No. 2, Pp. 124-130, 2017.

[3] A. P. Shukla, and M. Saini, "Moving Object Tracking of Vehicle Detection: A Concise Review," International Journal of Signal Processing, Image Processing and Pattern Recognition, Vol. 8, No.3, Pp. 169-176, 2015.

[4] S. K. Singh, and U. Sharma, "Simulink Model for Object Tracking Using Optical Flow," International Journal of Science and Research (IJSR), Vol. 4, No. 6, Pp. 2323-2326, 2015.

[5] D. R. Vinary, AND N. L. Kumar, "Object Tracking Using Background Subtraction Algorithm," International Journal of Engineering Research and General Science, Vol. 3, No. 1, 2015.

[6] P. D. Mahamuni, R. P. Patil, and H. S. Thakar, "Moving Object Detection Using Background Subtraction Algorithm Using Simulink," IJRET: International Journal of Research in Engineering and Technology, Vol. 03, No. 06, 2014.

[7] J. Scott, M. A. Pusateri, and D. Cornish, "Kalman Filter Based Video Background Estimation,"

[8] S. Aslani, and H. M. Nasab, "Optical Flow Based Moving Object Detection and Tracking for Traffic Surveillance," International Journal of Electrical, Computer, Energetic, Electronic and Communication Engineering, Vol. 7, No. 9, Pp. 1252-1256, 2013.

[9] R. Revathi, and M. Hemalatha, "Certain Approach of Object Tracking using Optical Flow Techniques, "International Journal of Computer Applications, Vol. 53, No. 8, Pp. 50-57, 2012.

[10]M. Himanshu, M. Dubasi, and R. S. Soumya, "Video Object Tracking Using Motion Estimation," Thesis, Roulkela: Postgraduate of National Institute of Technology, 2012.

[11] Mahali, M. Izzuddin, and A. Harjoko, "Moving Object Tracking Using Mean-Shift Method Using Scale Orientation Alterations,” Berkala MIPA, Vol. 24, No. 2, 2014. 